\title{
Apathy: Neurobiology, Assessment and Treatment
}

\author{
Mario Fahed, David C. Steffens \\ Department of Psychiatry, University of Connecticut, School of Medicine, Farmington, CT, USA
}

Apathy is a highly prevalent, disabling and treatment resistant syndrome. It is defined as a quantitative reduction of goaldirected activity in comparison to the patient's previous level of in multiple dimensions including behavior/cognition, emotion and social interaction. It has been described in major depressive disorder, Alzheimer's disease, frontotemporal dementia, Parkinson's Disease, cerebrovascular disease, and vascular dementia, among others. This review will address the neuropsychology and associated neurobiological underpinnings of apathy in the above conditions, identify specific methods to assess apathy clinically, and review the literature on managing apathy across these various disorders.

KEY WORDS: Apathy; Reward; Anhedonia.

\section{INTRODUCTION}

Apathy is a common symptom in older, cognitively impaired adults. In its first conceptualizations, apathy was described as a "lack of motivation, later evolving into a syndrome with multiple dimensions [1]. An expert panel recently reported revised criteria for apathy [2], defining it as a quantitative reduction of goal-directed activity in comparison to the patient's previous level of functioning (criterion A). Symptoms must persist for at least four weeks, and affect at least two of the three apathy dimensions (behavior/cognition; emotion; social interaction; criterion B). Apathy should cause identifiable functional impairments (criterion C), and should not be fully explained by other factors, such as effects of a substance or major changes in the patient's environment (criterion D).

Consistent with this definition, apathy should be differentiated from other common conditions such as depression and its core symptom of anhedonia. In their excellent review, Chong and Husain [3] emphasize that apathy, although debilitating, is consistently distinct from depression and generalized cognitive dysfunction.

Received: February 20, 2021 / Revised: March 9, 2021

Accepted: March 10, 2021

Address for correspondence: Mario Fahed

Department of Psychiatry, University of Connecticut, School of

Medicine, 263 Farmington Ave., Farmington, CT 06030, USA

E-mail: fahed@uchc.edu

ORCID: https://orcid.org/0000-0003-1509-1091
As a symptom, the prevalence of apathy in disorders that directly involve the cortex is $60 \%$, and disorders of subcortical structures developing apathy at a $40 \%$ rate [4]. It has been described in multiple disorders including Alzheimer's disease, frontotemporal dementia, Huntington's disease, major depressive disorder, Parkinson's Disease, schizophrenia, cerebrovascular disease, traumatic brain injury, and vascular dementia [5]. Moreover, a large population based study of behavioral and psychological symptoms of cognitive impairment found apathy to be highly prevalent at $31.9 \%$. The same study found it to be persistent and disabling [6].

In fact, apathy has been further been associated with functional decline, caregiver burden, increased cost of care due to early institutionalization, and increased mortality [7] . Apathy has also been described in healthy individuals with an estimated prevalence of $1.45 \%$ in young subjects with reduced quality of life and behavioral activation [8] and shows patterns of increasing with age, especially in persons above the age of 65 [9].

This review will focus on neurocognitive, biological and clinical aspects of apathy. We will discuss the neuropsychology and associated neurobiological underpinnings of apathy in various pathological conditions, identifiy methods to assess apathy clinically, and review the literature on managing apathy across various disorders.

(c) This is an Open-Access article distributed under the terms of the Creative Commons Attribution Non-Commercial License (http://creativecommons.org/licenses/by-nc/4.0) which permits unrestricted non-commercial use, distribution, and reproduction in any medium, provided the original work is properly cited. 


\section{APATHY AND THE REWARD SYSTEM ACROSS PATHOLOGIES}

Apathy has also been described as a failure to generate options for behavior; a diminished ability to select between options that depend on weighing their potential costs and benefits; and an impaired ability to initiate action and to learn from outcomes [10]. Another conceptualization of apathy details it as a multidimensional syndrome reflecting deficits in executive functioning (lack of motivation to plan, organize or sustain attention), emotional functioning (bunting, indifference) and initiation (impaired generation of thoughts) [11]. Throughout the literature, authors have described similar concepts with terms such as "initiative, enthusiasm, emotion" or "Intellectual curiosity, action initiation, self-awareness", leading up to the consensus criteria included above [11]. Although definitions of apathy and anhedonia can sometimes be conflated, Apathy presents as an overall lack of motivation based on decreased goal-directed behavior or thoughts, as well as affective flattening or indifference. [1]. Indeed, a lack of desire to pursue a reward or pleasure from a reward is common to both apathy and anhedonia, while emotional blunting/indifference is specific to apathy. An anhedonic individual may still consider social relationships as being important despite a lack of drive to pursue them while an apathetic individual no longer finds social relationships to be a valuable endeavor [12]. Another common concept commonly conflated with Apathy and

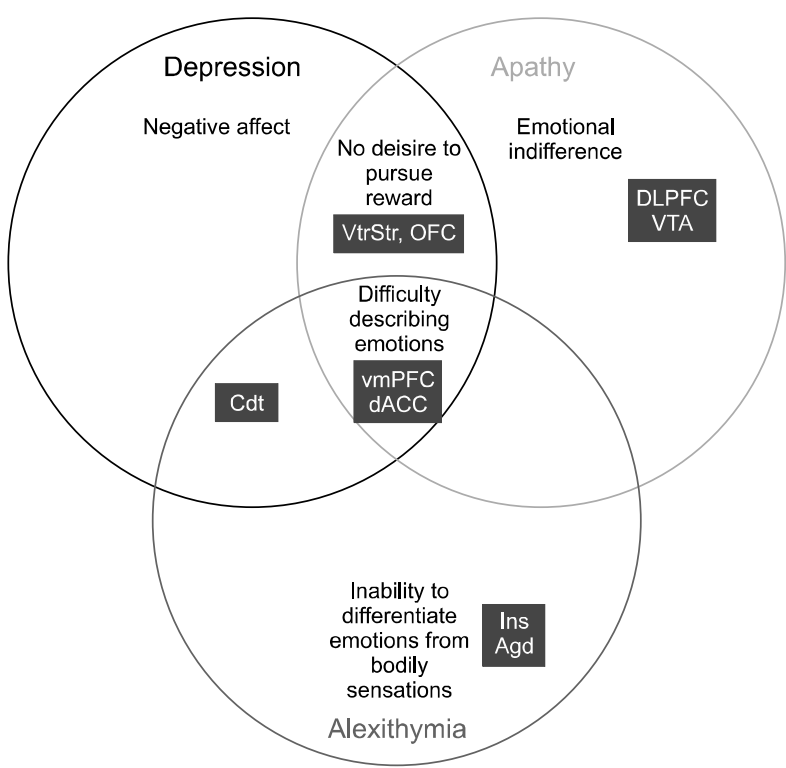

Anhedonia is Alexithymia, which refers to difficulty identifying and describing feelings often linked to functional somatic disorders [13] (Fig. 1).

From a neuroscience perspective, multiple attempts have been made to describe the underlying mechanisms of apathy. It was originally described as a dysfunction in the dopaminergic system leading to lack of motivation [1]. Across various pathologies, apathy seems associated with disruption of medial frontal cortex (Anterior Cingulate Cortex $[\mathrm{ACC}]$ and Orbitofrontal Cortex [OFC]), as well as subcortical structures (ventral striatum, medial thalamus, ventral tegmental area), with dorsal anterior cingulate cortex (dACC), and ventral striatum (VS) being the most consistently identified [14]. Other structures linked to apathy in Alzheimer's disease (both in decreased volume or decreased metabolism on functional magnetic resonance imaging $[\mathrm{fMRI}])$ are the posterior cingulate cortex, the putamen, and the inferior temporal cortex, with a greater association with right-sided compared to left-sided structures [15].

Other regions implicated less consistently in the goaldirected dimension of apathy are the insula, Dorsolateral Prefrontal Cortex (DLPFC), OFC, which are monosynaptically connected to each other and to the dACC and VS [14]. The dACC-VS circuit seems to have a role in the 3 components of motivation: willingness to initiate an action, to sustain performance of an action, and to learn whether actions are worth performing. The circuit plays a role in giving actions a value, when making a cost-benefit

- VtrStr: ventral striatum vmPFC: ventromedial prefrontral

OFC: orbitofrontal

cortex

dACC: dorsal anterior

cingulate cortex

Cdt: caudate

dLPFC: dorsolateral

prefrontal cortex

VTA: ventral tegmental

area

- Ins: insula

Agd: amygdala
Fig. 1. Broad clinical and neuroscientific distinctions between depression, apathy and alexithymia $[5,13]$. 
decision [14]. The value ascribed to performing action (reward anticipation) is independent from its "hedonic impact" or "liking" [16], which is deficient in anhedonia, a symptom of depression. Hedonic impact has been linked to activity in the medial orbitofrontal cortex (mOFC) [17] and the ventral striatum, the latter seems to code for positive prediction errors when reward is unexpected [18]. In fact, in studying patients with schizophrenia, Simon et al. concluded that apathy was observed with deficits in anticipatory reward processing (ventral striatum activity). This contrasts with anhedonia, which seems to be linked to a deficit in response to rewarding outcomes (ventral striatum and mOFC activity) [19].

\section{Neurobiology of Apathy in Late-life Depression}

Apathy in depression is a common finding particularly in depression with late-age onset [20]. Late-life depression (LLD) has been associated with several functional neural changes: an increase in metabolism in ventral limbic structures, including the amygdala, the pregenual anterior cingulate, the posterior orbital cortex, and the posterior cingulate and decreased metabolism in dorsal neocortical areas and basal ganglia, including the lateral and dorsolateral cortex, the dorsal anterior cingulate, and caudate nucleus [21].

Compared with elderly controls, older depressed adults showed increased intrinsic connectivity within the frontal parietal network (increased connectivity between superior parietal lobe [SPL] and DLPFC). The SPL appears to be involved in directing attention to visual and tactile stimuli as well as monitoring of internal body representation [22]. DLPFC on the other hand has been shown to have altered activity in preparation to overcome prepotency learning tasks, showing increased activation with increased preparatory demand $[23,24]$. Hypotheses about this include that older depressed individuals have increased attentional control for internal body states [22].

For patients with LLD, there is also increased intrinsic activity within the sensorimotor network, that is hypothesized to be linked to compensatory mechanisms counteracting cognitive decline [22] and within the default mode network including left precuneus, the subgenual ACC, and lateral parietal regions, the ventromedial prefrontal cortex (vmPFC) [21]. The latter has also been found to have attenuated intrinsic activity, which was positively associated with depression severity and normalized with treatment [25]. In fact, the vmPFC (which includes the orbitofrontal cortex, the rostral cingulate cortex, and the ventral medial frontal gyrus) has been associated with the evaluation and regulation of emotional events and stimuli and the contextual processing of reward. In LLD, there is a decreased response to negative compared with positive stimuli [25]. Others found decreased functional connectivity between the regions within the reward network: right nucleus accumbens and the right medial orbitofrontal cortex (mOFC), and between the regions within the attention network, right rostral ACC and bilateral superior frontal gyrus [23].

\section{Neurobiology of Apathy in Vascular Lesions}

Apathy is also seen as a common poststroke symptom [26]. In acute stroke, apathy may be the result of functional changes affecting an area involved in apathy generation. Instances of this include strokes in the ACC, striatum, ventral basal ganglia, thalamus and PFC. A remarkable finding in strokes specifically, is that apathy can result from damages to areas outside of those involved in apathy. This is theorized to be the consequence of diaschisis and transneuronal degeneration. Diaschisis is functional impairment due to distant damage to connected other parts of the brain. An example of this is reduced prefrontal hemodynamic response following subcortical, cerebellar and thalamic infarcts. Transneuronal degeneration are structural changes that follow these functional alterations [27]. Studies by Jang et al. [28] show that when left or right caudate lesions led to apathy by secondary lesions to the white matter tracts joining the caudate and prefrontral cortex. Further evidence of the role of white matter tracts in the genesis of apathy is found in Cerebral small vessel disease. Damage to major white matter tracts such as anterior cingulum has been associated with apathy [29]. Whitespread damage to network efficacy seems to follow damage from small vessel disease [30].

\section{Neurobiology of Apathy in Neurocognitive Disorders}

In Mild Cognitive Impairment, Apathy is an early symptom and its presence has been linked to rapid progression of symptoms, and a 7-fold increase in the rate of convertsion to Alzheimer's disease (AD) [31].

In Alzheimer's, Apathy is one of the most frequent symptom found on the neuropsychiatric inventory (NPI), 
with an overall prevalence of 49\% [10,32].

A disconnection syndrome involving microalterations of the anterior cingulum (AC) is a proposed mechanism explaining the onset of apathy in $\mathrm{AD}$ [33]. The posterior cingulate cortex, the putamen, and the inferior temporal cortex, with a greater association with right-sided compared to left-sided structures are linked to Alzheimer's both in decreased volume or decreased metabolism on fMRI [15]. As detailed in Nobis and Husain's [10] excellent review, there was no consensus between studies about the regional atrophy and apathy in Alzheimer's. Abnormal white matter integrity on the other hand in AD patients has been reported. These white matter changes were found in the corpus callosum, which was also correlated with severity of apathy. Severity of apathy was correlated with loss of integrity of the right superior longitudinal fasciculus, left anterior and posterior cingulum, and bilateral uncinate fasciculus. On beta Amyloid PET imaging, severity of apathy was correlated with beta Amyloid deposition in medial and orbitofrontal areas, insula, and right ACC [10].

In Frontotemporal Dementia, Apathy is especially prevalent in the behavioral variant with a frequency as high as $90.5 \%$. It is the most common neuropsychiatric symptom and is prevalent throughout the course of the illness $[7,34]$. Apathy has been correlated with changes in the orbitofrontal cortex and anterior cingulate, particularly on the right, with atrophy noted on morphometry MRI in the right lateral orbitofrontal cortex, anterior cingulate [35]. PET studies have shown correlation between apathy and hypometabolism of orbitofrontal cortex and anterior cingulate [36].

In Parkinson's Disease and Lewy body dementia, the prevalence of apathy has ranged from $17 \%$ to $70 \%$ and $35 \%$ to $100 \%$ [37].

The neuroscientific pathways underlying apathy in Parkinson's Disease pertain to a dopaminergic deficit in the mesocorticolimbic pathway. PET studies show decreased dopamine binding in the Orbitofrontal cortex, amygdala, dorsolateral prefrontal cortex and striatum. When a ligand that binds to both dopamine and noradrenaline transporters was used, the degree of apathy correlated inversely with binding in the ventral striatum. When executive dysfunction predominates in apathy rather than the emotional blunting, lesions affecting this are dorsolateral and ventrolateral prefrontal cortex or the caudate [38].

\section{ASSESSMENT OF APATHY}

To diagnose Apathy, the 2018 expert panel recommends the criteria detailed in Table 1.

Because Apathy tends to be subtle and manifest differently, we recommend the use of scales for its assessment and tracking. Choosing the tool used to assess and track severity can vary based on the pathology, the informant (clinician, patient, caretaker) and the symptoms [2]. The supplement to the above consensus criteria contains an excellent and exhaustive repository of all the developed tools. In our experience, the most used tool is the Apathy Evaluation Scale (AES). It is based on Marin's conceptualization of apathy, which described a triad of cognition, behavior and emotion. Alternatively, Levy and Dubois identified three apathetic subtypes: cognitive, emotional-affective and auto-activation. The latter component is what distinguishes it from Marin's conceptualization [39]. Another widely used too is the dimensional apathy scale which allows for examination and grading of the subtypes above [39]. Although apathy and depression can present with overlapping symptoms, the emotional-affective subtype is different from depression in that its "emotional neutrality" whereas depression has a negative affect [39].

In Parkinson's, the Lille apathy rating scale (LARS) is a sensitive screen tool capable of distinguishing between apathy and depression [40]. The Apathy-motivation Index (AMI) was adapted from the LARS to assess apathy in healthy people [41].

\section{MANAGEMENT OF APATHY}

Apathy can be a devastating symptom for both the patient and their caregivers. Patients with apathy have a lower quality of life, have increased risk of mortality and early institutionalization [4]. Caregivers may view the loss of motivation as volitional or oppositional behavior, leading to additional caregiver distress [42]. Careful and repeated psychoeducation is necessary.

From a non-pharmacological standpoint, although the quality of the evidence varies, several interventions were found to be effective. In their systematic review, Theleritis et al. [43] found evidence for recreational activities derived from the Need-driven dementia-compromised be- 
Table 1. 2018 Consensus panel diagnostic criteria for Apathy

Criterion A: A quantitative reduction of goal-directed activity either in behavioral, cognitive, emotional or social dimensions in comparison to the patient's previous level of functioning in these areas. These changes may be reported by the patient himself/herself or by observation of others.

Criterion B: The presence of at least 2 of the 3 following dimensions for a period of at least four weeks and present most of the time B1. Behaviour \& Cognition:

Loss of, or diminished, goal-directed behaviour or cognitive activity as evidenced by at least one of the following:

- General level of activity: the patient has a reduced level of activity either at home or work, makes less effort to initiate or accomplish tasks spontaneously, or needs to be prompted to perform them.

- Persistence of activity: He/she is less persistent in maintaining an activity or conversation, finding solutions to problems or thinking of alternative ways to accomplish them if they become difficult.

- Making choices: He/she has less interest or takes longer to make choices when different alternatives exist (e.g., selecting TV programs, preparing meals, choosing from a menu, etc.)

- Interest in external issue: He/she has less interest in or reacts less to news, either good or bad, or has less interest in doing new things.

- Personal wellbeing: He/she is less interested in his/her own health and wellbeing or personal image (general appearance, grooming, clothes, etc.).

B2. Emotion

Loss of, or diminished, emotion as evidenced by at least one of the following:

- Spontaneous emotions: the patient shows less spontaneous (self-generated) emotions regarding their own affairs, or appears less interested in events that should matter to him/her or to people that he/she knows well.

- Emotional reactions to environment: He/she expresses less emotional reaction in response to positive or negative events in his/her environment that affect him/her or people he/she knows well (e.g., when things go well or bad, responding to jokes, or events on a TV program or a movie, or when disturbed or prompted to do things he/she would prefer not to do).

- Impact on others: He/she is less concerned about the impact of his/her actions or feelings on the people around him/her.

- Empathy: He/she shows less empathy to the emotions or feelings of others (e.g., becoming happy or sad when someone is happy or sad, or being moved when others need help).

- Verbal or physical expressions: He/she shows less verbal or physical reactions that reveal his/her emotional states.

B3. Social interaction

Loss of, or diminished engagement in social interaction as evidenced by at least one of the following:

- Spontaneous social initiative: the patient takes less initiative in spontaneously proposing social or leisure activities to family or others.

- Environmentally stimulated social interaction: He/she participates less, or is less comfortable or more indifferent to social or leisure activities suggested by people around him/her.

- Relationship with family members: He/she shows less interest in family members (e.g., to know what is happening to them, to meet them or make arrangements to contact them).

- Verbal interaction: He/she is less likely to initiate a conversation, or he/she withdraws soon from it

- Homebound: He/she prefer to stays at home more frequently or longer than usual and shows less interest in getting out to meet people.

Criterion C: These symptoms $(\mathrm{A}-\mathrm{B})$ cause clinically significant impairment in personal, social, occupational, or other important areas of functioning.

Criterion D: The symptoms $(\mathrm{A}-\mathrm{B})$ are not exclusively explained or due to physical disabilities (e.g., blindness and loss of hearing), to motor disabilities, to a diminished level of consciousness, to the direct physiological effects of a substance (e.g., drug of abuse, medication), or to major changes in the patient's environment.

Adapted from the article of Robert et al. (Eur Psychiatry 2018;54:71-76) [2].

havior model, live music, Snoezelen (multi-sensory room), reminiscence therapy, and stimulation-retreat model of care, among others. There is a death of evidence for the treatment of isolated apathy in healthy individuals.

\section{Management of Apathy in Depression}

There are multiple reports of Selective Serotonin Reuptake Inhibitors (SSRIs) worsening apathy in depression. Yuen et al. [44] found that apathy is a common feature of late-life depression but is a distinct clinical syndrome that is "insufficiently addressed by SSRI alone". In fact, they found that individuals with structural abnormalities of the posterior subgenual cingulate cortex which connects via the uncinate fasciculus to limbic structures were more likely to show persistence of apathy in geriatric depression. They also found that improvement of apathy with SSRI was independent of change in depression scores. There were no statistically significant worsening of apathy scores in this study, though this may in part due to its limitations [44]. 


\section{Management of Apathy in Cerebrovascular Disease}

Given the heterogeneity of the presentation, data lacks as to effective treatments in vascular apathy. A study by Moretti et al. [45], found long-term rivastigmine use in subcortical vascular dementia to be tolerable and correlated with stability of cognitive performance and daily function, with improvements in executive function, planning behavior, neuropsychiatric symptoms and social conduct. Although apathy is not specifically mentioned, the above improvements are all components of apathy [45].

\section{Management of Apathy in Alzheimer's Disease}

Cholinesterase inhibitors have been used for the treatment of Apathy in AD. Evidence for this is lacking as most reviews have found either no or limited evidence of beneficial benefit $[10,46]$. Promising preliminary results were found when donepezil was combined with the cholinergic precursor choline alphoscerate (versus donepezil alone). It demonstrated significantly lower ratings of apathy after 1 and 2 years in the combined treatment arm compared to donepezil-only [47]. Methylphenidate has also been studied in mild Alzheimer's disease and was found to improve apathy over 12-weeks. It also improved cognition, functional status, caregiver burden, CGI scores (improvement and severity), and depression [48]. Subsequent reviews concluded that methylphenidate's effect was small but significant, though it should be weighed against its significant side effects including high blood pressure, cough and osteoarticular pain [46]. In the Okayama Depression and Apathy Project, patients receiving escitalopram showed significant improvement in GDS score in Alzheimer's whereas patients receiving sertraline showed improvement in the Apathy Scale [49].

Repetitive Transcranial Magnetic Stimulation (rTMS) targeting frontal lobe dysfunction (abnormal activity in DLPFC, OFC, medial prefrontal cortices [mPFC], anterior cingulate gyrus, and supplementary motor area [SMA]) with $10 \mathrm{~Hz}$ on the left DLPFC produced the best effect size in a crossover study conducted with nine subjects with $\mathrm{MCl}$. Improvements in AES-S compared to sham groups were noticed at 2 weeks. They also saw improvements in cognition. Though the sample size was a limiting factor, this study could open the door to further studies to utilize rTMS for the treatment of apathy in $\mathrm{MCl}$ [31].

\section{Management of Apathy in Parkinson Pathologies}

Pramipexole was found to produce significantly lower total apathy scores, especially when compared with ropinirole and L-dopa [50]. Overvliet et al. [51] recommend, based on an extensive review of literature, rivastigmine as a treatment of apathy in patients with Parkinson's Disease, and pramipexole or selegiline in Parkinson's Disease patients with apathy without cognitive disturbance. It bears mentioning however that rivastigmine was not effective in controlling apathy in Parkinson's Disease dementia on a 1-year follow-up [52]. Pagonabarraga and colleagues recommended determining the predominant subdomain of apathy in PD patients may help guide the choice of therapy: dopamine agonists and other dopaminergic drugs, such as methylphenidate, for emotional-affective deficits; AcetylCholinesterase Inhibitors for cognitive deficits; and dopamine agonists for auto-activation deficit [38].

Depression, a very common comorbidity of Parkinson's Disease, is often treated with SSRIs. SSRIs have been linked to worsening of apathy in Parkinson's Disease [53]. Serotonin and Norepinephrine Reputake Inhibitors, quetiapine and clozapine were not associated with apathy worsening, while Selegiline and Rasagiline were associated with lower prevalence of apathy [53].

\section{Management of Apathy in Lewy Body Disease}

There is limited evidence for the use of Cholinesterase inhibitors in randomized control trials. Evidence for pramipexole is promising, though lacking studies in which apathy is the primary outcome measure.

\section{Management of Apathy in FTD}

A small study where agomelatine, an antidepressant with MT1 and MT2 receptor agonism and 5- $\mathrm{HT}_{2 \mathrm{C}}$ receptor antagonism, was compared to melatonin, agomelatine showed significant reduction of apathy scores in patients with FTD, and associated decrease in caregiver distress. Agomelatine presumably leads to an increase in dopaminergic and noradrenergic tone in the prefrontal cortex [54]. Citalopram was associated with a decrease in apathy in a study by Herrmann et al. [55]. Intranasal oxytocin is currently under investigation in a phase 2 trial by Finger et al. [56]. 


\section{CONCLUSIONS}

Apathy is a prevalent and debilitating symptom that can occur in several degenerative and non-degenerative pathologies. Its neuroscientific underpinnings involve the dopaminergic system and several cortical and subcortical structures including ACC, OFC, DLPFC, ventral striatum, medial thalamus, ventral tegmental area, among others.

Although recognized as largely treatment resistant, the literature describes several non-pharmacological interventions [43].

From a transdiagnostic lens, pharmacological interventions should be tailored to the apathy subtype identified using the Dimensional Apathy Scale. Pagonabarraga et al. [38] present a reasonable framework to approach psychpharmacology in Apathy. For deficiencies in reward valuation (emotional blunting), dopamine receptor agonists, methylphenidate and bupropion may be used. For deficits in executive dysfunction (decreased interest, cognitive blunting), utilizing acetylcholinesterase inhibitors is recommended. For deficits in autoactivation (lack of self-initiated behaviors which respond to external stimuli), dopamine receptor agonists are indicated [38]. If depression (negative affect, hopelessness, guilt, pessimism) coexists with apathy, non-SSRI antidepressants are recommended as a first line as SSRI may worsen apathy when depressive symptoms are comorbid [38].

\section{- Conflicts of Interest}

No potential conflict of interest relevant to this article was reported.

\section{Author Contributions}

Conceptualization and Writing: Mario Fahed and David C. Steffens.

\section{ORCID}

Mario Fahed https://orcid.org/0000-0003-1509-1091

David C. Steffens https://orcid.org/0000-0001-6626-7398

\section{REFERENCES}

1. Marin RS. Apathy: a neuropsychiatric syndrome. J Neuropsychiatry Clin Neurosci 1991;3:243-254.

2. Robert $\mathrm{P}$, Lanctôt $\mathrm{KL}$, Agüera-Ortiz L, Aalten $\mathrm{P}$, Bremond $\mathrm{F}$, Defrancesco $\mathrm{M}$, et al. Is it time to revise the diagnostic criteria for apathy in brain disorders? The 2018 international con- sensus group. Eur Psychiatry 2018;54:71-76.

3. Chong TT, Husain M. The role of dopamine in the pathophysiology and treatment of apathy. Prog Brain Res 2016;229:389426.

4. van Reekum R, Stuss DT, Ostrander L. Apathy: why care? J Neuropsychiatry Clin Neurosci 2005;17:7-19.

5. Husain M, Roiser JP. Neuroscience of apathy and anhedonia: a transdiagnostic approach. Nat Rev Neurosci 2018;19:470484.

6. van der Linde RM, Matthews FE, Dening T, Brayne C. Patterns and persistence of behavioural and psychological symptoms in those with cognitive impairment: the importance of apathy. Int J Geriatr Psychiatry 2017;32:306-315.

7. Massimo L, Evans LK. Differentiating subtypes of apathy to improve person-centered care in frontotemporal degeneration. J Gerontol Nurs 2014;40:58-65.

8. Pardini M, Cordano C, Guida S, Grafman J, Krueger F, Sassos $\mathrm{D}$, et al. Prevalence and cognitive underpinnings of isolated apathy in young healthy subjects. J Affect Disord 2016;189: 272-275.

9. Brodaty H, Altendorf A, Withall A, Sachdev P. Do people become more apathetic as they grow older? A longitudinal study in healthy individuals. Int Psychogeriatr 2010;22:426-436.

10. Nobis L, Husain M. Apathy in Alzheimer's disease. Curr Opin Behav Sci 2018;22:7-13.

11. Radakovic R, Abrahams S. Multidimensional apathy: evidence from neurodegenerative disease. Curr Opin Behav Sci 2018;22:42-49.

12. Rizvi SJ, Pizzagalli DA, Sproule BA, Kennedy SH. Assessing anhedonia in depression: potentials and pitfalls. Neurosci Biobehav Rev 2016;65:21-35.

13. Kano M, Fukudo S. The alexithymic brain: the neural pathways linking alexithymia to physical disorders. Biopsychosoc Med 2013;7:1.

14. Le Heron C, Apps MAJ, Husain M. The anatomy of apathy: a neurocognitive framework for amotivated behaviour. Neuropsychologia 2018;118(Pt B):54-67.

15. Huey ED, Lee S, Cheran G, Grafman J, Devanand DP. Brain regions brain regions involved in arousal and reward processing are associated with apathy in Alzheimer's disease and frontotemporal dementia. J Alzheimers Dis 2017;55:551-558.

16. Gard DE, Kring AM, Gard MG, Horan WP, Green MF. Anhedonia in schizophrenia: distinctions between anticipatory and consummatory pleasure. Schizophr Res 2007;93: 253-260.

17. Kringelbach ML. The human orbitofrontal cortex: linking reward to hedonic experience. Nat Rev Neurosci 2005;6:691702.

18. Berns GS, McClure SM, Pagnoni G, Montague PR. Predictability modulates human brain response to reward. I Neurosci 2001;21:2793-2798.

19. Simon JJ, Biller A, Walther S, Roesch-Ely D, Stippich C, Weisbrod $\mathrm{M}$, et al. Neural correlates of reward processing in schizo- 
phrenia--relationship to apathy and depression. Schizophr Res 2010;118:154-161.

20. Steffens DC, Krishnan KR. Structural neuroimaging and mood disorders: recent findings, implications for classification, and future directions. Biol Psychiatry 1998;43:705-712.

21. Alexopoulos GS. Frontostriatal and limbic dysfunction in late-life depression. Am J Geriatr Psychiatry 2002;10:687-695.

22. Cieri F, Esposito R, Cera N, Pieramico V, Tartaro A, di Giannantonio M. Late-life depression: modifications of brain resting state activity. J Geriatr Psychiatry Neurol 2017;30: 140-150.

23. Tadayonnejad R, Yang S, Kumar A, Ajilore O. Multimodal brain connectivity analysis in unmedicated late-life depression. PLoS One 2014;9:e96033.

24. Aizenstein HJ, Butters MA, Wu M, Mazurkewicz LM, Stenger VA, Gianaros PJ, et al. Altered functioning of the executive control circuit in late-life depression: episodic and persistent phenomena. Am J Geriatr Psychiatry 2009;17:30-42.

25. Brassen S, Kalisch R, Weber-Fahr W, Braus DF, Büchel C. Ventromedial prefrontal cortex processing during emotional evaluation in late-life depression: a longitudinal functional magnetic resonance imaging study. Biol Psychiatry 2008;64: 349-355.

26. Starkstein SE, Fedoroff JP, Price TR, Leiguarda R, Robinson RG. Apathy following cerebrovascular lesions. Stroke 1993; 24:1625-1630.

27. Tay J, Lisiecka-Ford DM, Hollocks MJ, Tuladhar AM, Barrick TR, Forster A, et al. Network neuroscience of apathy in cerebrovascular disease. Prog Neurobiol 2020;188:101785.

28. Jang $\mathrm{SH}$, Kim SH, Kwon HG. Injury of the prefronto-caudate tract in a patient with apathy following intracerebral hemorrhage in the caudate nucleus. Acta Neurol Belg 2019;119: 143-145.

29. Hollocks MJ, Lawrence AJ, Brookes RL, Barrick TR, Morris RG, Husain $\mathrm{M}$, et al. Differential relationships between apathy and depression with white matter microstructural changes and functional outcomes. Brain 2015:138(Pt 12):3803-3815.

30. Tuladhar AM, Tay J, van Leijsen E, Lawrence AJ, van Uden IWM, Bergkamp M, et al. Structural network changes in cerebral small vessel disease. I Neurol Neurosurg Psychiatry 2020;91:196-203.

31. Padala PR, Padala KP, Lensing SY, Jackson AN, Hunter CR, Parkes CM, et al. Repetitive transcranial magnetic stimulation for apathy in mild cognitive impairment: a double-blind, randomized, sham-controlled, cross-over pilot study. Psychiatry Res 2018;261:312-318.

32. Lyketsos CG, Steinberg M, Tschanz JT, Norton MC, Steffens DC, Breitner JC. Mental and behavioral disturbances in dementia: findings from the Cache County Study on Memory in Aging. Am J Psychiatry 2000;157:708-714.

33. Kim JW, Lee DY, Choo IH, Seo EH, Kim SG, Park SY, et al. Microstructural alteration of the anterior cingulum is associated with apathy in Alzheimer disease. Am/ Geriatr Psychiatry
2011;19:644-653.

34. Mendez MF, Lauterbach EC, Sampson SM. An evidencebased review of the psychopathology of frontotemporal dementia: a report of the ANPA Committee on Research. J Neuropsychiatry Clin Neurosci 2008;20:130-149.

35. Rosen HJ, Allison SC, Schauer GF, Gorno-Tempini ML, Weiner MW, Miller BL. Neuroanatomical correlates of behavioural disorders in dementia. Brain 2005;128(Pt 11): 2612-2625.

36. Sarazin M, Michon A, Pillon B, Samson Y, Canuto A, Gold G, et al. Metabolic correlates of behavioral and affective disturbances in frontal lobe pathologies. J Neurol 2003;250:827833.

37. Liu J, Cooper CA, Weintraub D, Dahodwala N. Pharmacological treatment of apathy in Lewy body disorders: a systematic review. Parkinsonism Relat Disord 2019;60:14-24.

38. Pagonabarraga J, Kulisevsky J, Strafella AP, Krack P. Apathy in Parkinson's disease: clinical features, neural substrates, diagnosis, and treatment. Lancet Neurol 2015;14:518-531.

39. Radakovic R, Abrahams S. Developing a new apathy measurement scale: Dimensional Apathy Scale. Psychiatry Res 2014;219:658-663.

40. Sockeel P, Dujardin K, Devos D, Denève C, Destée A, Defebvre L. The Lille apathy rating scale (LARS), a new instrument for detecting and quantifying apathy: validation in Parkinson's disease. J Neurol Neurosurg Psychiatry 2006;77: 579-584.

41. Ang YS, Lockwood P, Apps MA, Muhammed K, Husain M Distinct subtypes of apathy revealed by the apathy motivation index. PLoS One 2017;12:e0169938.

42. Theleritis C, Politis A, Siarkos K, Lyketsos CG. A review of neuroimaging findings of apathy in Alzheimer's disease. Int Psychogeriatr 2014;26:195-207.

43. Theleritis C, Siarkos K, Politis AA, Katirtzoglou E, Politis A. A systematic review of non-pharmacological treatments for apathy in dementia. Int J Geriatr Psychiatry 2018;33:e177e192.

44. Yuen GS, Gunning FM, Woods E, Klimstra SA, Hoptman MJ, Alexopoulos GS. Neuroanatomical correlates of apathy in late-life depression and antidepressant treatment response. J Affect Disord 2014;166:179-186.

45. Moretti R, Torre P, Antonello RM, Cazzato G, Bava A. Rivastigmine in subcortical vascular dementia: an open 22-month study. J Neurol Sci 2002;203-204:141-146.

46. Rea R, Carotenuto A, Fasanaro AM, Traini E, Amenta F. Apathy in Alzheimer's disease: any effective treatment? ScientificWorldlournal 2014:2014:421385.

47. Rea R, Carotenuto A, Traini E, Fasanaro AM, Manzo V, Amenta F. Apathy treatment in Alzheimer's disease: interim results of the ASCOMALVA trial. J Alzheimers Dis 2015;48: 377-383.

48. Padala PR, Padala KP, Lensing SY, Ramirez D, Monga V, Bopp $\mathrm{MM}$, et al. Methylphenidate for apathy in community-dwell- 
ing older veterans with mild Alzheimer's disease: a doubleblind, randomized, placebo-controlled trial. Am J Psychiatry 2018;175:159-168.

49. Takemoto M, Ohta Y, Hishikawa N, Yamashita T, Nomura E, Tsunoda K, et al. The efficacy of sertraline, escitalopram, and nicergoline in the treatment of depression and apathy in Alzheimer's disease: the Okayama Depression and Apathy Project (ODAP). J Alzheimers Dis 2020;76:769-772.

50. Pérez-Pérez J, Pagonabarraga J, Martínez-Horta S, FernándezBobadilla R, Sierra S, Pascual-Sedano B, et al. Head-to-head comparison of the neuropsychiatric effect of dopamine agonists in Parkinson's disease: a prospective, cross-sectional study in non-demented patients. Drugs Aging 2015;32:401407.

51. Overvliet GM, Vlaar A, de Haan C, Lampe I. Pharmacological treatment of apathy in Parkinson's disease, review of the literature. Parkinsonism Relat Disord 2020;79(Suppl 1):E70E71.

52. Moretti R, Caruso P, Dal Ben M. Rivastigmine as a symptomatic treatment for apathy in Parkinson's dementia complex: new aspects for this riddle. Parkinsons Dis 2017;2017: 6219851.

53. Zahodne LB, Bernal-Pacheco O, Bowers D, Ward H, Oyama $\mathrm{G}$, Limotai N, et al. Are selective serotonin reuptake inhibitors associated with greater apathy in Parkinson's disease? I Neuropsychiatry Clin Neurosci 2012;24:326-330.

54. Callegari I, Mattei C, Benassi F, Krueger F, Grafman J, Yaldizli Ö, et al. Agomelatine improves apathy in frontotemporal dementia. Neurodegener Dis 2016;16:352-356.

55. Herrmann N, Black SE, Chow T, Cappell J, Tang-Wai DF, Lanctôt KL. Serotonergic function and treatment of behavioral and psychological symptoms of frontotemporal dementia. Am J Geriatr Psychiatry 2012;20:789-797.

56. Finger E, Berry S, Cummings J, Coleman K, Hsiung R, Feldman $\mathrm{HH}$, et al. Adaptive crossover designs for assessment of symptomatic treatments targeting behaviour in neurodegenerative disease: a phase 2 clinical trial of intranasal oxytocin for frontotemporal dementia (FOXY). Alzheimers Res Ther 2018;10: 102. 